Antarctic ${ }^{14} \mathrm{C}$ levels are especially low, posing problems when dating marine material. Age corrections up to 1,400 years are necessary for recent material, whereas around 400 years is typical elsewhere 5 .

We obtained age estimates for the brachiopod Liothyrella uva from Signy Island, Antarctica $\left(60^{\circ} 43^{\prime} \mathrm{S}, 45^{\circ} 36^{\prime} \mathrm{W}\right)$, by counting alpha growth checks in shell valves $\left(G_{\mathrm{c}}\right)$ and from measured increments $\left(G_{i}\right)$ in a 2-year mark-recapture growth study. The $G_{\mathrm{i}}$ ages were 1.84 times older than $G_{\mathrm{c}}$ values ( $a$ in the figure), and this factor was significantly different from $2(t=15.8, P<0.001)$. Ten brachiopods were then aged using $G_{\mathrm{c}} \times 1.84$, and samples were cut from the oldest parts of their shell valves. Shell samples thus obtained were deposited between 1945 and 1985 , and contained enhanced ${ }^{14} \mathrm{C}$ from the mid-1950s onwards ( $b$ in the figure). The ${ }^{14} \mathrm{C}$ increase occurred within $1-2$ years of the first radiocarbon bomb signals found in nearby Antarctic peninsula ice-cores ${ }^{6}$. The ${ }^{14} \mathrm{C}$ signals peaked around 1962 and declined thereafter, although another peak possibly occurred between 1975 and 1980, following enhanced ice-core activity in 1972-74 (ref. 6).

Shell-activity data are, therefore, 2-5 years later than terrestrial signals, peaks are less precise, and the time required for the signal to return to background levels is much longer. This reflects the large carbon pool and long residence time in Antarctic circumpolar water, and contradicts the hypothesis that upwelling deep water with low radiocarbon content causes reduced signals in polar oceans ${ }^{3}$, which would cause signals to decay more rapidly (upwelling is also unlikely from oceanographic considerations - Signy lies within the strongly stratified Weddell-Scotia confluence ${ }^{7}$ ). Lower marine signals in polar latitudes are, therefore, more probably due to reduced atmospheric ${ }^{14} \mathrm{C}$ supply and long-term radioisotope incorporation in ice.

The close correlation of shell age with ${ }^{14} \mathrm{C}$ signal confirms that growth checks were not formed annually, and indicates either entrainment to a biennial environmental cycle that occasionally breaks down, or an endogenous cycle with a periodicity of less than 2 years. Using the $\times 1.84$ conversion of growth rings to age gives a maximum of 52 years for L. uva (2 years' periodicity gives 56 years). Temperate and tropical brachiopod ages are generally $2-15$ years. Ages $>50$ years were calculated recently for Magellania fragilis ${ }^{2}$, a Weddell Sea species, assuming annual growth checks. Periodicities approximating 2 years would predict ages of $90-100$ years.

Sea-ice dynamics in the Weddell Sea have a 7-9-year cycle ${ }^{8}$. Poor phytoplankton years also occurred at Signy Island on four occasions between 1972 and 1982 (ref. 9). However, these were not at approximately 2-yearly intervals, and no known sea-ice cycle approximates 2 years. Other longterm cycles identified include 4-6-year periodicities in life-history traits of phocids around the Antarctic Peninsula, which were suggested as being related to the $\mathrm{El}$ Niño-Southern Oscillation. However, the trends in these data were weak, and different biological cycles occurred in different phases with the El Niño signal ${ }^{10}$. A clear environmental signal of shorter periodicity has yet to be identified in this area. Alter-

\begin{abstract}
a, Brachiopod shell length compared with age from external shell check-marks $\left(G_{c}\right)$ and from growth increments $\left(G_{i}\right)$ from a field mark-recapture study. Shell check-marks were analysed twice in 113 individuals by both authors independently (size range, 3-55 mm length; total, $n=205$ )
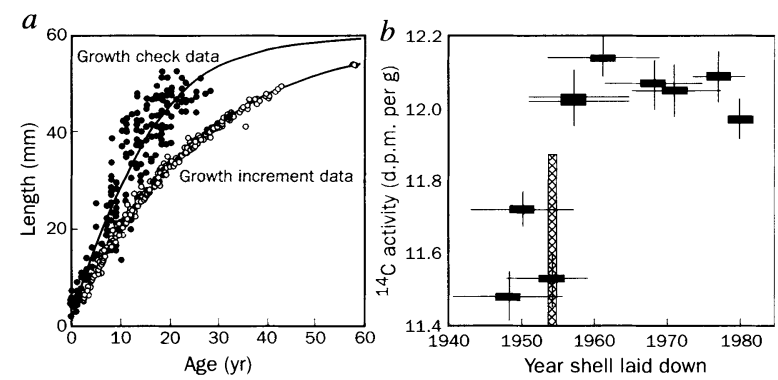

For $G_{i}$ data, $286 \mathrm{~L}$. uva (size range: 4-54 mm length) were collected, measured and returned to the collection site. They were remeasured after $2 \mathrm{yr}$. General von Bertalanffy functions were fitted to both data sets $\left(G_{\mathrm{c}}\right.$ data: $K=0.086, D=1.582, t_{0}=-1.834, R^{2}=0.873 ; G_{i}$ data: $K=0.040, D=0.991, R^{2}=0.996 ; L_{\infty}$ was set to $60.0 \mathrm{~mm}$ to cover the whole size range) using the SIMPLEX iterative nonlinear fitting algorithm. The difference between the two functions fitted a linear model (age $\left(G_{i}\right)=-0.785+1.842 \times$ age $\left(G_{c}\right) ; r^{2}=0.997 ; n=113$ ). After removing handling effects, by comparison with 6-month-duration trials (data not shown) at the same locality, mortality in the $G_{i}$ experiment was $<2 \% \mathrm{yr}^{-1}$. Growth rates in shorterduration trials were also not significantly different from the 2 -yr experiment. $b$, Shell ${ }^{14} \mathrm{C}$ activity (decompositions $\mathrm{min}^{-1} \mathrm{~g}^{-1}, \pm$ s.d.) versus age. The oldest $5-8 \mathrm{~mm}$ (corresponding to approximately the first 3-4 yr of life, calculated from $G_{i}$ data in a) of the brachial valves of 10 L. uva 12-57 mm long were analysed by accelerated mass spectroscopy. They had 8-25 growth rings (15-46 yr old, using the $\times 1.84$ factor). Horizontal bars, $3-4-y r$ period of shell deposition; horizontal lines, $95 \%$ confidence interval for sample ages; hatched vertical bar, earliest enhanced radioisotope signals in Antarctic Peninsula ice-cores. ${ }^{14} \mathrm{C}$ data for samples before the ice-core signal were significantly lower than later ones (ANOVA, $F=76.5 ; 2,6$ d.f.; $P<0.001)$. Shell-activity levels correspond to ${ }^{14} \mathrm{C}$ ages of $890-1,340 \mathrm{yr}$.
\end{abstract}

natively, approximately biennial growth checks could result from endogenous cycles. Several Antarctic benthic invertebrates have reproductive cycles of $>1$ year (ref. 11), and reduced overall resource supply may underpin such extended cycles. Clearly, care is needed in interpreting shell check-marks and ageing cold water species, without independent dating. Whether the underlying causes of near-biennial growth bands are taxon-specific or environmentally modulated also requires elucidation.

Lloyd S. Peck

Natural Environment Research Council,

British Antarctic Survey, High Cross,

Madingley Road, Cambridge CB3 OET, UK

Thomas Brey

Alfred Wegener Institute for Polar and

Marine Research,

D-2850 Bremerhaven, Germany

1. Peck, L. S. et al. Treatise on Invertebrate Palaeontology (H), Brachiopods Ch. III (Geol. Soc. Am. \& Univ. Kansas, Lawrence, in the press).

2. Brey, T. et al. J. mar. Biol. Ass. U.K. 75, 857-869 (1995).

3. Broecker, W. S. \& Peng, T. S. Nature 356, 587-589 (1992).

4. Weidman, C. R. \& Jones, G. A. J. geophys. Res. 98, 14577-14588 (1993).

5. Gordon, J. E. \& Harkness, D. D. Quat. Sci. Rev. 11, 697-708 (1992).

6. Peel, D. A. \& Clausen, H. B. J. Glaciol. 28, 43-55 (1982).

7. Muench, R. D., Gunn, J. T. \& Husby, D. M. J. geophys. Res. 95, 18177-18190 (1990).

8. Murphy, E. J. et al. Deep Sea Res. 42, 1045-1062 (1995).

9. Clarke, A., Holmes, L. J. \& White, M. G. Br. Antarct. Surv. Bull. 80, 65-86 (1988).

10. Ward Testa, J. et al. Can. J. Fish. aquat. Sci. 48 , 631-639 (1991).

11. Clarke, A. Invert. Reprod. Dev. 22, 176-184 (1992).

\section{Mating success of male birds}

SIR - Widemo and Owens' examined the amount of variation in mating success on mating arenas (leks) among male birds of the ruff Philomachus pugnax, and concluded that the skew in male mating success decreases with increasing average lek size. Such a relationship has important implications for our understanding of the evolution of lekking as a mating system and sexual selection in general ${ }^{1-3}$.

Widemo and Owens' conclusion was challenged by Mackenzie et $a l^{2}$ on the basis that the skew index ${ }^{4,5}$ used to quantify the amount of variation in mating success between males is expected to decrease with lek size under a null version of the model in which mating occurs entirely at random. Although it is true that the skew index decreases with increased lek size under random mating (dashed line, $a$ in the figure), this decrease is much less pronounced than the exponential decrease suggested by Mackenzie et $a l^{2}{ }^{2}$ (solid line, $a$ in the figure). The drop in skew observed by Widemo and Owens in larger leks is therefore not simply an artefact of the relationship between the skew index and lek size.

Two other possible factors could 


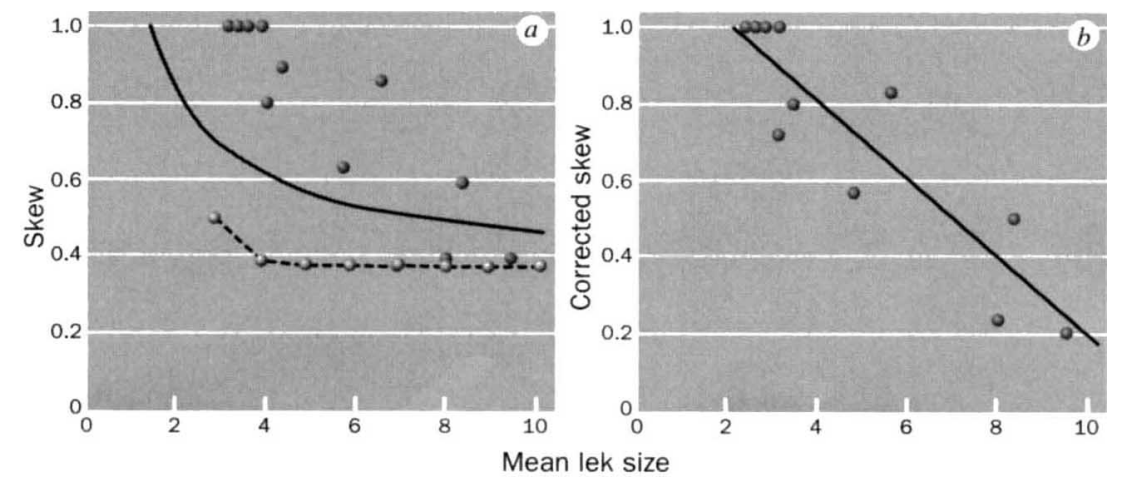

a, Observed relationship across leks between mean lek size and skew in male success. The mating skew index is calculated as the ratio of observed to maximum skew ${ }^{3.4}$, which is becoming a commonly used measure of heterogeneity in reproductive success among group members (for example, refs $6-8$ ). The dashed line is the expected relationship when females select males randomly (the null model). The expected distribution assumes that the average mating success of males is unity. Solid line, expected relationship proposed in ref. 2 under the hypothesis of random mating. The discrepancy is due to an error in ref. 2, partly caused by a problem with the random number generator (A. Mackenzie, personal communication) and most importantly by the inclusion of lek sizes smaller than two while estimating the effect of lek size variation on skew. Skew should not be estimated for leks smaller than two, and indeed, at least two males were always present when females visited leks (F. Widemo, personal communication). b, Observed relationship between mean lek size and 'corrected' skew when controlling for the effect of average mating success and the interdependence of the skew index on the number of males $\left(r^{2}=0.83 ; n=11 ; P<0.0001\right)$. Expected skew under random mating (random skew) was estimated for each of the 11 leks by considering the number of males and females visiting a lek. The corrected skew quantifies the difference between the observed skew and the expected skew under random mating: corrected skew = (observed skew random skew)/ (maximum skew - random skew), where maximum skew $=1$. A computer program for calculating the expected skew under the hypothesis of random mating (for leks with variable number $(>2$ ) of males and females) is available on request from the authors (e-mail: Ikeller@ulys.unil.ch).

explain the relationship observed by Widemo and Owens under the null hypothesis of random mating. First, greater skew is expected to characterize smaller leks if these were visited more frequently by males spending only a little time on the lek. This suggestion predicts a negative association between lek size and the average proportion of time that males are at the lek site. However, the correlation between these two variables is not significant $\left(r^{2}=0.02, n=11, P>0.05\right.$; data provided by Widemo and Owens) and the sign of the correlation is positive, indicating that variation in the time that males spend at leks of different sizes is not responsible for the observed relationship between mating skew and lek size.

Second, as Mackenzie et al. ${ }^{2}$ correctly point out, the skew index is sensitive to the mean mating success. As with any other formula for calculating skewness, the skew index tends to decrease with increasing mean mating success. Therefore, if mean male mating success increases with increasing lek size, we expect a negative relationship between skew and lek size even if mating is random.

To test this hypothesis, we examined the relationship between mean lek size and mating skew, while controlling for the effects of variation in mean male mating success and the interdependence of the skew index on the number of males (data provided by Widemo and Owens). For each of the 11 leks, we estimated the expected skew under the null hypothesis of random mating and quantified the deviation between this value and the observed skew (corrected skew; $b$ in the figure). This analysis reveals a strong and significant negative relationship between the corrected skew and mean lek size.

In conclusion, Mackenzie et $a l^{2}$ are right to point out that the skew index can be affected by factors such a lek size and mean mating success, even under the null hypothesis of matings occurring entirely at random. However, our analysis demonstrates that these factors do not account for the relationship that Widemo and Owens describe, thus validating the conclusion that mating skew decreases with lek size in the ruff.

\section{Laurent Keller*}

\section{Michael J. B. Krieger}

Institut de Zoologie et d'Ecologie Animale, University of Lausanne, BB,

\section{Lausanne, Switzerland}

1. Widemo, F. \& Owens, I. P. F. Nature 373, 148-151 (1995).

Mackenzie, A. et al. Nature 336, 471 (1995)

3. Höglund, J. \& Alatalo, R. V. Leks (Princeton Univ. Press, 1995).

4. Keller, L. \& Vargo, E. L. in Queen Number and Sociality in Insects (ed. Keller, L.) 16-44 (Oxford Univ. Press, 1993).

5. Reeve, H. K. \& Ratnieks, F. L. W. in Queen Number and Sociality in Insects (ed Keller, L) 45-85 (Oxford Univ. Press, 1993)

6. Keller, L. \& Reeve, H. K. Trends Ecol. Evol. 9, 98-102 (1994).

. Bourke, A. F. G. \& Heinze, J. Phil. Trans. R. Soc. B345, 359-372 (1994).

8. Reeve, H. K. \& Keller, L. Am. Nat 145, 119-132 (1995).

*Also at Zoologisches Institut, Bern University, Ethologische Station Hasli, 3032 Hinterkappelen, Switzerland.

\section{Reconstruction of museum specimens}

SIR - Museum collections of fishes, amphibians and reptiles have traditionally been repositories for single, fluid-fixed specimens (holotypes) on which most type-descriptions are based. These specimens are generally unsuitable for molecular studies because they have been fixed in formalin. Their value could be greatly enhanced by the use of noninvasive imaging techniques to provide structural information for the study of taxonomy, myology, functional morphology and also new characters for phylogenetic studies.

With the advent of high-resolution magnetic resonance imaging (MRI) scanners and reduction in the cost of scanning ( $£ 150$ per specimen for the studies reported here), it is now feasible to use these methods for soft-tissue studies of smaller zoological specimens. As examples, we have used preserved elasmobranch fishes imaged using a high-resolution (4.7-T) scanner with a $20-\mathrm{mT} \mathrm{m}^{-1}$ gradient set. Our comparisons between preserved and fresh material show that formalin fixation itself has no effect on image characteristics.

Standard, non-invasive techniques (radiography, whole-body staining) can give only limited anatomical information, because of the low radiographic contrast of the wholly cartilaginous, loosely articulated skeleton and the logistical difficulties of staining these large fishes, especially such multi-articulated structures as the branchial arches. In addition, neither technique can reveal details of myology, which can be studied only by dissection. Elasmobranch hyaline cartilage is friable and easily deformed during maceration, as osteocytes are absent and structural apatite mineralization is superficial except in the vertebral centra of some orders ${ }^{1}$.

Counts of vertebrae are important at the specific level of elasmobranch taxonomy. There have been difficulties in the X-ray determination of vertebral counts ${ }^{2}$. Notochordal sharks' are deep-sea sharks with reduced calcification, which prevents accurate radiographic determination of complete counts. In situ enumeration of vertebral centra was investigated with twodimensional MRI imaging. A representative section of the anterior vertebral column of the bramble shark Echinorhinus brucus is shown in Fig. $1 a$ overleaf. Segmentation of the notochord occurs internally, although there is little external evidence for it in the notochordal sheath. This is the first presentation of external and internal structure of the spinal column of $E$. brucus, and shows that vertebral counts using MRI are now feasible in these elasmobranchs.

Using the ANALYZE software ${ }^{3}$, threedimensional reconstruction from two- 